\title{
Pharmaceutical Quality/CMC Manufacturing Site Unique Identifier Terminology
}

National Cancer Institute

\section{Source}

National Cancer Institute. Pharmaceutical Quality/CMC Manufacturing Site Unique

Identifier Terminology. NCI Thesaurus. Code C133859.

A category of terminology used to qualify the information pertaining to manufacturing site unique identifier types in the framework of the Pharmaceutical Quality/Chemistry, Manufacturing and Controls documents. 